\title{
Development of quality metrics for ambulatory pediatric cardiology: Chest pain
}

\author{
Jimmy C. Lu MD ${ }^{1}$ () | Manish Bansal MBBS ${ }^{2}$ | Sarina K. Behera MD³ \\ Jeffrey R. Boris MD ${ }^{4}$ | Brian Cardis MD ${ }^{5}$ | John S. Hokanson MD ${ }^{6}$ | \\ Bahram Kakavand $\mathrm{MD}^{7}$ | Roy Jedeikin MD, MBA ${ }^{8}$
}

${ }^{1}$ Department of Pediatrics and Communicable Diseases, University of Michigan, Ann Arbor, Michigan, USA

${ }^{2}$ Department of Pediatrics, Texas Children's Hospital, Houston, Texas, USA

${ }^{3}$ Stanford Children's Health at California Pacific Medical Center, San Francisco, California, USA

${ }^{4}$ Department of Pediatrics, University of Pennsylvania, Philadelphia, Pennsylvania, USA

${ }^{5}$ Department of Pediatrics, Emory University, Atlanta, Georgia, USA

${ }^{6}$ Department of Pediatrics, University of Wisconsin, Madison, Wisconsin, USA

${ }^{7}$ Department of Pediatrics, Nemours Children's Hospital, Orlando, Florida, USA

${ }^{8}$ Arizona Pediatric Cardiology, Phoenix Children's Hospital, Phoenix, Arizona, USA

\section{Correspondence}

Jimmy C. Lu, University of Michigan Congenital Heart Center, C.S. Mott Children's Hospital, 1540 East Hospital Drive, Ann Arbor, MI 48109-4204. Email: jimmyl@umich.edu

\begin{abstract}
Objective: As part of the American College of Cardiology Adult Congenital and Pediatric Cardiology Section effort to develop quality metrics (QMs) for ambulatory pediatric practice, the chest pain subcommittee aimed to develop QMs for evaluation of chest pain.

Design: A group of 8 pediatric cardiologists formulated candidate QMs in the areas of history, physical examination, and testing. Consensus candidate QMs were submitted to an expert panel for scoring by the RAND-UCLA modified Delphi process. Recommended QMs were then available for open comments from all members.

Patients: These QMs are intended for use in patients 5-18 years old, referred for initial evaluation of chest pain in an ambulatory pediatric cardiology clinic, with no known history of pediatric or congenital heart disease.

Results: A total of 10 candidate QMs were submitted; 2 were rejected by the expert panel, and 5 were removed after the open comment period. The 3 approved QMs included: (1) documentation of family history of cardiomyopathy, early coronary artery disease or sudden death, (2) performance of electrocardiogram in all patients, and (3) performance of an echocardiogram to evaluate coronary arteries in patients with exertional chest pain.

Conclusions: Despite practice variation and limited prospective data, 3 QMs were approved, with measurable data points which may be extracted from the medical record. However, further prospective studies are necessary to define practice guidelines and to develop appropriate use criteria in this population.
\end{abstract}

KEYWORDS

chest pain, quality metrics, pediatric cardiology

\section{1 | INTRODUCTION}

Chest pain is a common referral diagnosis for ambulatory pediatric cardiology evaluation. ${ }^{1-3}$ However, the majority of these patients do not have cardiac causes of chest pain. ${ }^{2-4}$ In a cohort of 3700 patients with median follow-up of 4.4 years, a cardiac cause was identified in only 37 cases (1\%), with no cardiac deaths. ${ }^{2}$ Unfortunately, there are few prospective studies and no validated practice guidelines to inform practice, and children presenting with a complaint of chest pain may undergo extensive evaluation with significant practice variation. ${ }^{5}$

The American College of Cardiology (ACC) Adult Congenital and Pediatric Cardiology Council (ACPC) recognized the need to develop quality metrics (QMs) in 5 key areas of pediatric and congenital heart disease. ${ }^{6}$ The Chest Pain subcommittee aimed to develop QMs for evaluation of chest pain in children. This article reviews the process of quality metric development, a summary of candidate and approved QMs, and a discussion of key issues that arose during the process. 


\section{METHODS}

The team consisted of 8 pediatric cardiologists from the United States, including a team leader. Beginning in September 2012, initial conference calls discussed the scope of the project, with identification of key areas for evaluation. The patient population was identified as patients ages 5-18 years, seen for an initial consultation at an ambulatory pediatric cardiology clinic with a chief complaint of chest pain. Candidate QMs were not intended to encompass patients with a previous diagnosis of acquired or congenital heart disease or patients presenting to an emergency room or inpatient setting.

Three categories were identified: history, physical examination, and testing. The team was then divided into subgroups of 2-3 cardiologists, each with a subgroup lead, to evaluate the existing literature and to formalize candidate QMs within their respective category. The QMs included a description of the proposed metric, numerator, denominator, and exclusions. All resulting QMs were discussed at conference calls involving the entire team. QMs were evaluated for clinical relevance, ease of measurement, and feasibility for widespread application.

The formalized QMs were subsequently evaluated by an expert panel from the ACC ACPC section using the RAND-UCLA modified Delphi process ${ }^{7}$ in two rounds of voting (first, individually, and second, in an in-person meeting). ${ }^{6}$ Metrics were scored for validity and feasibility on a scale of 1 to 9, with 9 having the highest validity and feasibility. QMs with a mean validity 7 to 9 and median feasibility 4 to 9 were approved. Approved QMs were then posted on the ACC website for a 4-week open comment period, with comments solicited from all members. All comments were reviewed by the expert panel, for final approval of QMs.

\section{3 | RESULTS}

\section{1 | Literature review}

Although there are no validated practice guidelines, a Standardized Clinical Assessment and Management Plan (SCAMP) for chest pain was developed at Boston Children's Hospital, ${ }^{4}$ which has been shown to decrease practice variation and resource utilization, ${ }^{4,5}$ and has been extended to other centers in the region. ${ }^{8}$ This algorithm utilizes elements of history (exertional chest pain with no alternative explanation, concerning past medical or family history), abnormal physical exam, or abnormal electrocardiogram to determine which patients should undergo further evaluation with echocardiography. The SCAMP has not been endorsed by a governing organization, and differs from clinical practice guidelines in that data are analyzed on a recurring basis, allowing for ongoing revision of the SCAMP.

Guidelines not specific to chest pain were also considered. The American Heart Association (AHA) Scientific Statement on cardiovascular risk in high-risk pediatric patients includes family history of early coronary artery disease for risk stratification. ${ }^{9}$ In addition, the AHA Scientific Statement on preparticipation sports evaluation recommends evaluation of family history for hypertrophic cardiomyopathy, dilated cardiomyopathy, and sudden cardiac death. ${ }^{10}$
The existing literature on chest pain in children consists largely of retrospective cohort studies. Although chest pain is a common indication for referral to a pediatric cardiologist, cardiac causes are rare. ${ }^{2-4,11}$ Among patients with cardiac conditions which could cause chest pain, the most common diagnosis was a coronary artery anomaly, with $70 \%$ presenting with exercise-induced chest pain. ${ }^{11}$ Echocardiography is the test of choice for initial evaluation of coronary artery anomalies. ${ }^{12}$ The electrocardiogram can be abnormal in cardiac causes of chest pain, ${ }^{11,13,14}$ and has a high negative predictive value for hypertrophic cardiomyopathy, long QT syndrome, and Wolff-Parkinson-White syndrome. ${ }^{15}$ However, rhythm recording devices (RRD; such as Holter monitors and event recorders) have not demonstrated benefit in patients with isolated chest pain. ${ }^{4,16}$ Exercise stress test (EST) for chest pain is typically negative, ${ }^{1,2,4,17,18}$ and can even be negative in patients with documented coronary artery anomalies. ${ }^{19,20}$

\section{$3.2 \mid \mathrm{QM}$}

Consensus was reached for 10 candidate QMs (Table 1), with 3 based on history, 1 on physical examination, and 6 on testing. Due to the rarity of positive findings, improved quality care and cost effectiveness may be reflected in the absence rather than in the performance of further testing. Thus, instead of measuring the proportion of patients who received an appropriate test or documentation, 3 candidate QMs (4, 6, and 7) evaluated the proportion of patients who had testing performed under inappropriate conditions. For these measures, optimal care would be reflected in a lower rather than higher percentage of tests performed. However, in the absence of accepted appropriate use criteria, there was concern in the group that adoption of such QMs may be premature, and could be interpreted as establishing new guidelines.

After the RAND process, two history-based metrics (history of fever and history of Kawasaki disease) did not pass due to inadequate validity. Validity and feasibility scores were previously reported. ${ }^{6}$

The highest attrition of QMs occurred after the open comments and final review by the steering committee, during which 5 of 8 metrics were rejected. The physical examination QM (palpation of the chest wall) was rejected due to comments that it was not a reliable method to exclude cardiac disease. Although this maneuver is commonly used, reproducible pain to palpation does not exclude the possibility of an additional cause of cardiac chest pain, such as myocarditis. Similarly, QMs 4, 6, and 7, intended to evaluate for inappropriate testing, were rejected due to concerns for overlap of symptoms in this age group (eg, having both musculoskeletal chest pain as well as cardiac pathology). These QMs could potentially limit physicians in pursuing additional testing which may be appropriate in the context of other clinical signs and symptoms. In addition, QMs evaluating exercise treadmill test were deferred, due to comments regarding the ambiguity of exercise stress testing (eg, electrocardiogram or echocardiogram). Stress echocardiography may be a useful adjunct in evaluation of exertional chest pain in some patients. Due to the ambiguity and potential controversy, these QMs thus could not yet be approved as indicators of quality care.

Thus, after the RAND process and open comments period, 3 QMs were approved for children ages $5-18$ years with an initial presentation 
TABLE 1 Candidate QM for chest pain submitted to expert panel

\section{Candidate measure}

\section{Family history}

Numerator: Patients with documentation of positive or negative family history for cardiomyopathy, early coronary artery disease in a first-degree relative (male $<55$ years, female $<65$ years), or sudden cardiac death

Denominator: Patients 5-18 years old seen in ambulatory pediatric cardiology clinic with chief complaint of chest pain

Exclusions: None

\section{Palpation of the chest wall}

Numerator: Patients with documentation of palpation of the chest (eg, whether chest pain is reproducible) in the physical exam

Denominator: Patients 5-18 years old seen in ambulatory pediatric cardiology clinic with chief complaint of chest pain

Exclusions: None

\section{Electrocardiogram}

Numerator: Patients who had an ECG performed on the day of consultation or within one month of initial consultation for chest pain

Denominator: Patients 5-18 years old seen in ambulatory pediatric cardiology clinic with chief complaint of chest pain

Exclusions: None

\section{No echocardiogram in pediatric patients with musculoskeletal chest pain}

Numerator: Patients that had an echocardiogram performed or ordered on the day of the cardiology clinic visit

Denominator: Patients 5-18 years old seen in ambulatory pediatric cardiology clinic with clinical evidence of musculoskeletal chest wall pain (reproducible to palpation over the costochondral junctions, chest wall, or under the costal margins)

Exclusions: Patients with exertional chest pain component, family history of sudden death or cardiomyopathy, or abnormal physical examination or electrocardiogram findings

\section{Echocardiogram for exertional chest pain}

Numerator: Patients that had an echocardiogram performed or ordered on day of cardiology clinic visit that includes a comment regarding coronary artery anatomy

Denominator: Patients 5-18 years old seen in ambulatory pediatric cardiology clinic for chief complaint of chest pain, with solely exertional chest pain (eg, documentation of chest pain with exercise, but not at rest)

Exclusions: Previous echocardiogram or cardiac magnetic resonance or computed tomography within 6 months with documentation of coronary artery anatomy, or chest pain characteristic of musculoskeletal chest pain or exercise-induced asthma

\section{Appropriate use of RRD for chest pain}

\section{Numerator: Patients that had an RRD ordered}

Denominator: Patients 5-18 years old seen in ambulatory pediatric cardiology clinic with chief complaint of isolated chest pain

Exclusions: Previous diagnosis of cardiomyopathy or arrhythmias

\section{Utilization of EST in musculoskeletal chest pain}

Numerator: Patients that had an EST performed or ordered on the day of the cardiology clinic visit

Denominator: Patients 5-18 years old seen in ambulatory pediatric cardiology clinic with clinical evidence of musculoskeletal chest wall pain (reproducible to palpation over the costochondral junctions, chest wall, or under the costal margins)

Exclusions: Patients with exertional chest pain component, family history of sudden death or cardiomyopathy, or abnormal physical examination or electrocardiogram findings

\section{Appropriate use of EST in patients with exertional chest pain}

\section{Numerator: Patients with documentation in HPI or ROS of exertional chest pain or associated palpitations or syncope}

Denominator: Patients 5-18 years old seen in ambulatory pediatric cardiology clinic for chief complaint of chest pain who had an EST performed or ordered on the day of evaluation

Exclusions: Patients with exertional chest pain component, family history of sudden death or cardiomyopathy, or abnormal physical examination or electrocardiogram findings

\section{History of fever}

Numerator: Patients with documentation of presence/absence of fever within the prior 30 days

Denominator: Patients 5-18 years old seen in ambulatory pediatric cardiology clinic with chief complaint of chest pain

Exclusions: None

\section{History of Kawasaki disease}

Numerator: Patients with documentation of presence/absence of a history of Kawasaki disease

Denominator: Patients 5-18 years old seen in ambulatory pediatric cardiology clinic with chief complaint of chest pain

Exclusions: None

of chest pain to an outpatient pediatric cardiology clinic: (1) evaluation of family history, (2) electrocardiogram in all patients, and (3) echocardiogram for exertional chest pain.

\section{4 | DISCUSSION}

This process led to successful development of $3 \mathrm{QM}$ for outpatient evaluation of chest pain in children and adolescents. This is the first
ACPC quality metric effort to evaluate a symptom (chest pain), rather than a diagnosis.

The frequency of chest pain referrals and broad variation of testing patterns make chest pain in children an essential area for standardization and improved quality of care. However, addressing a symptom rather than an established diagnosis raises difficulties in the breadth of patients and presentations. In children, symptoms may overlap significantly. For example, although fever in the appropriate context may suggest myocarditis, a potential cardiac cause of chest pain, it may be very 
nonspecific due to the frequency of viral infections in this population, and thus has insufficient validity to recommend as a quality metric. In addition, although further testing in many cases of musculoskeletal chest pain may be unnecessary, pain on palpation may not exclude an additional diagnosis, and there may be other extenuating historical or physical examination findings which would make additional testing reasonable. The lack of established guidelines or appropriate use criteria limits the ability to develop QMs that would help identify and evaluate clinical practice variation. Development of such guidelines or appropriate use criteria can be challenging since chest pain is a symptom rather than a diagnosis. However, future efforts to establish clinical standards and guidelines would be necessary to allow for additional QMs in the future.

The recommended QM offer discrete and measurable data points which can be extracted from the medical record, and should be documented in an appropriate evaluation of pediatric chest pain. The current work differs from existing literature, as these metrics are designed to be used as indicators of high quality patient care, rather than as an algorithm or practice guideline. They are intended for use retrospectively to assess adherence rather than prospectively to guide practice. Thus, although several candidate QMs addressed aspects of care which would typically be recommended, such as relevant history or palpation for reproducible musculoskeletal chest pain, the metrics could only include elements most closely aligned with existing published data, and which can be easily measured retrospectively from appropriate documentation.

There are limitations in this process. Although these metrics measure data points that are necessary for quality care, they are not sufficient. They are necessarily narrow, as the dearth of accepted guidelines and evidence prevents further reaching metrics of accepted quality. These metrics are not intended to form a broader clinical practice guideline or to address all potential testing for any given patient. The practitioner is still encouraged to assess each patient as an individual. The QMs are intended to evaluate clinical practice, not to refrain from ordering tests that may be appropriate for a specific patient or clinical scenario.

In conclusion, QM were developed for initial outpatient evaluation of chest pain in 5- to 18 -year-old patients. Family history of cardiomyopathy, early coronary artery disease or sudden death should be documented, electrocardiogram should be performed in all patients, and an echocardiogram should be performed to evaluate coronary arteries in patients with exertional chest pain. Further prospective research is necessary to acquire more data as well as to define practice guidelines and to develop appropriate use criteria in this population. As guidelines and appropriate use criteria are created and validated, further measures of quality care may be recommended in future efforts.

\section{ACKNOWLEDGMENTS}

The authors would like to thank Devyani Chowdhury, MD, Michelle Gurvitz, MD, and Ariane Marelli, MD, for their help and input.

\section{CONFLICT OF INTEREST}

The authors have no relationships relevant to the contents of this paper to disclose.

\section{AUTHOR CONTRIBUTIONS}

Drafting article: Lu

Concept/design: Lu, Bansal, Behera, Boris, Cardis, Hokanson, Kakavand, Jedeikin

Critical revision of article: Bansal, Behera, Boris, Cardis, Hokanson, Kakavand, Jedeikin

\section{ORCID}

Jimmy C. Lu MD iD http://orcid.org/0000-0002-2544-7402

\section{REFERENCES}

[1] Danduran MJ, Earing MG, Sheridan DC, Ewalt LA, Frommelt PC. Chest pain: characteristics of children/adolescents. Pediatr Cardiol. 2008;29(4):775-781.

[2] Saleeb SF, Li WY, Warren SZ, Lock JE. Effectiveness of screening for life-threatening chest pain in children. Pediatrics. 2011;128(5): e1062-e1068.

[3] Hanson CL, Hokanson JS. Etiology of chest pain in children and adolescents referred to cardiology clinic. WMJ. 2011;110(2):58-62.

[4] Friedman KG, Kane DA, Rathod RH, et al. Management of pediatric chest pain using a standardized assessment and management plan. Pediatrics. 2011;128(2):239-245.

[5] Verghese GR, Friedman KG, Rathod RH, et al. Resource utilization reduction for evaluation of chest pain in pediatrics using a Novel Standardized Clinical Assessment and Management Plan (SCAMP). J Am Heart Assoc. 2012;1(2). pii: jah3-e000349.

[6] Chowdhury D, Gurvitz M, Marelli A, et al. Development of quality metrics in ambulatory pediatric cardiology. J Am Coll Cardiol. 2017; 69(5):541-555.

[7] Normand SL, McNeil BJ, Peterson LE, Palmer RH. Eliciting expert opinion using the Delphi technique: identifying performance indicators for cardiovascular disease. Int J Qual Health Care. 1998;10(3): 247-260.

[8] Angoff GH, Kane DA, Giddins N, et al. Regional implementation of a pediatric cardiology chest pain guideline using SCAMPs methodology. Pediatrics. 2013;132(4):e1010-e1017.

[9] Kavey RE, Allada V, Daniels SR, et al. Cardiovascular risk reduction in high-risk pediatric patients: a scientific statement from the American Heart Association Expert Panel on Population and Prevention Science; the Councils on Cardiovascular Disease in the Young, Epidemiology and Prevention, Nutrition, Physical Activity and Metabolism, High Blood Pressure Research, Cardiovascular Nursing, and the Kidney in Heart Disease; and the Interdisciplinary Working Group on Quality of Care and Outcomes Research: endorsed by the American Academy of Pediatrics. Circulation. 2006;114(24):2710-2738.

[10] Maron BJ, Thompson PD, Ackerman MJ, et al. Recommendations and considerations related to preparticipation screening for cardiovascular abnormalities in competitive athletes: 2007 update: a scientific statement from the American Heart Association Council on Nutrition, Physical Activity, and Metabolism: endorsed by the American College of Cardiology Foundation. Circulation. 2007;115(12): 1643-1455. 
[11] Kane DA, Fulton DR, Saleeb S, Zhou J, Lock JE, Geggel RL. Needles in hay: chest pain as the presenting symptom in children with serious underlying cardiac pathology. Congenit Heart Dis. 2010;5(4):366-373.

[12] Davis JA, Cecchin F, Jones TK, Portman MA. Major coronary artery anomalies in a pediatric population: incidence and clinical importance. J Am Coll Cardiol. 2001;37(2):593-597.

[13] Ratnapalan S, Brown K, Benson L. Children presenting with acute pericarditis to the emergency department. Pediatr Emerg Care. 2011;27(7):581-585.

[14] Drossner DM, Hirsh DA, Sturm JJ, et al. Cardiac disease in pediatric patients presenting to a pediatric ED with chest pain. Am J Emerg Med. 2011;29(6):632-638.

[15] Rodday AM, Triedman JK, Alexander ME, et al. Electrocardiogram screening for disorders that cause sudden cardiac death in asymptomatic children: a meta-analysis. Pediatrics. 2012;129(4):e999-1010.

[16] Saarel EV, Stefanelli CB, Fischbach PS, Serwer GA, Rosenthal A, Dick M. Transtelephonic electrocardiographic monitors for evaluation of children and adolescents with suspected arrhythmias. Pediatrics. 2004;113(2):248-251. 2nd.

[17] Kyle WB, Macicek SL, Lindle KA, Kim JJ, Cannon BC. Limited utility of exercise stress tests in the evaluation of children with chest pain. Congenit Heart Dis. 2012;7(5):455-459.
[18] Nudel DB, Diamant S, Brady T, Jarenwattananon M, Buckley BJ, Gootman N. Chest pain, dyspnea on exertion, and exercise induced asthma in children and adolescents. Clin Pediatr. 1987;26(8): 388-392.

[19] Basso C, Maron BJ, Corrado D, Thiene G. Clinical profile of congenital coronary artery anomalies with origin from the wrong aortic sinus leading to sudden death in young competitive athletes. J Am Coll Cardiol. 2000;35(6):1493-1501.

[20] Frommelt PC, Sheridan DC, Berger S, Frommelt MA, Tweddell JS. Ten-year experience with surgical unroofing of anomalous aortic origin of a coronary artery from the opposite sinus with an interarterial course. J Thorac Cardiovasc Surg. 2011;142(5): 1046-1051.

How to cite this article: Lu JC, Bansal M, Behera SK, et al. Development of quality metrics for ambulatory pediatric cardiology: Chest pain. Congenital Heart Disease. 2017;12:751-755. https://doi.org/10.1111/chd.12509 